\title{
Directly Deflecting Particle Dark Matter
}

\author{
Asher Berlin®, Raffaele Tito D’Agnolo, Sebastian A. R. Ellis, Philip Schuster, and Natalia Toro \\ SLAC National Accelerator Laboratory, 2575 Sand Hill Road, Menlo Park, California 94025, USA
}

(Received 29 August 2019; published 2 January 2020)

\begin{abstract}
We propose a new strategy to directly detect light particle dark matter that has long-ranged interactions with ordinary matter. The approach involves distorting the local flow of dark matter with time-varying fields and measuring these distortions with shielded resonant detectors. We apply this idea to sub-MeV dark matter particles with very small electric charges or coupled to a light vector mediator, including the freezein parameter space targeted by low mass direct detection efforts. This approach can probe dark matter masses ranging from $10 \mathrm{MeV}$ to below a meV, extending beyond the capabilities of existing and proposed direct detection experiments.
\end{abstract}

DOI: 10.1103/PhysRevLett.124.011801

Dark matter (DM) constitutes the majority of matter in the Universe, but an understanding of its nature and interactions remains elusive. The landscape of viable candidates is vast, ranging in mass from $\sim 10^{-22} \mathrm{eV}$ to superplanetary scales, with a broad variety of possible interactions. Moreover, DM may have particlelike (particlenumber-conserving) or fieldlike (linear-in-DM-field and hence, particle-number-violating) interactions. These two classes of interaction can occur in overlapping mass ranges, but give rise to very different phenomenology. This wide range of possibilities motivates employing a varied set of detection strategies (see Ref. [1] for an overview).

Searches for sub-eV DM often assume interactions linear in the DM field and exploit the semiclassical properties of the coherent DM field for detection. Furthermore, these searches often rely on detectors that are resonantly matched to the angular frequency of the oscillating DM field (set by the DM constituent mass). Searches for heavier fieldlike DM, up to keV-scale masses, exploit the absorption of DM particles by electrons or nuclei through these single-field couplings. On the other hand, detection strategies for DM with an exact (or approximate) DM-particle-number symmetry rely on single-particle scattering reactions. These setups can only observe DM heavy enough that a single scattering event transfers an observably large energy to the detector. As a result, such experiments are most sensitive in the $10 \mathrm{GeV}-\mathrm{TeV}$ mass range, but recent technological advances are enabling sensitivity to $\mathrm{MeV}$-scale DM scattering and may eventually reach keV mass thresholds [1].

Published by the American Physical Society under the terms of the Creative Commons Attribution 4.0 International license. Further distribution of this work must maintain attribution to the author(s) and the published article's title, journal citation, and DOI. Funded by SCOAP ${ }^{3}$.
In this work, we propose a new approach ("direct deflection") to search for sub-MeV particlelike DM that exploits long-range interactions between DM and ordinary matter, as expected in prominent freeze-in scenarios and any model of DM with an ultralight force mediator. The approach is based on inducing collective effects in the DM fluid on detector lengthscales that can leave a measurable trace in resonant detectors. In the collectiveness of the effect, and in its enabling technologies, direct deflection is reminiscent of light fieldlike DM searches. However, the signal does not depend on DM behaving like a classical field (large occupation number per cubic de Broglie wavelength, with linear coupling to matter), but instead on the far laxer condition that it behaves like a classical fluid (large number of DM particles in the volume of the apparatus, with numberconserving interactions). In models that can be probed by both direct deflection and traditional direct detection experiments, the deflection approach has a parametric advantage at low DM masses, where the low energy deposited by singleparticle scattering becomes difficult to measure but collective effects of the DM fluid are enhanced.

The general direct deflection concept can be realized in multiple ways, potentially targeting a variety of motivated DM models. Here, we focus on a concrete setup that is sensitive to DM particles coupled to a light kinetically mixed vector mediator. One motivation for exploring such models is so-called "freeze-in" DM that arises through very feeble interactions between DM and standard model (SM) matter [2]. Notably, for keV-MeV DM masses, freeze-in through a very light mediator is among the very few models known to have a viable and predictive cosmology while being plausibly explorable with terrestrial experiments. This scenario has become an important benchmark for low-mass direct detection experiments [1], and exhibits terrestrial phenomenology akin to millicharged particles.

With this motivation in mind, our proposal is to induce and subsequently detect oscillating effective DM millicharge or 
millicurrent densities, using large driven electromagnetic fields and well-shielded resonant detectors, similar to "light-shining-through-a-wall"-type experiments [3-5]. Unlike such experiments, however, our setup does not rely on the production of new light states. Instead, ambient DM from the Milky Way halo passing through an oscillating electromagnetic field is deflected, setting up propagating waves of DM millicharge and millicurrent. These DM waves can penetrate a downstream electromagnetic shield by virtue of the tiny DM coupling (see, e.g., Fig. 2), establishing small oscillating electric and magnetic fields that can be measured with a resonator coupled to a sensitive magnetometer. A schematic illustration of this "wind-blowing-through-a-wall" apparatus is shown in Fig. 1. This technique is based on tested technology, complements and competes with other direct detection proposals in the $\mathrm{keV}-\mathrm{MeV}$ mass range, and is sensitive to much smaller masses, going beyond current astrophysical constraints for DM lighter than a keV. The anticipated reach of various experimental configurations is shown in Fig. 2.

Millicharged and millicharge-like dark matter.-To illustrate our idea, we consider the scenario in which DM, denoted by $\chi$, couples to standard electromagnetism with an effective charge $q_{\text {eff }} \ll 1$. This is often referred to as "millicharged" DM, and in its simplest incarnation requires no new particles beyond the DM itself. A natural way for such effective models to arise is when the DM is charged under a hidden sector gauge boson $A_{\mu}^{\prime}$ (a "dark photon") that kinetically mixes with the SM photon [15],

$$
\mathcal{L} \supset \frac{\epsilon}{2} F_{\mu \nu} F^{\prime \mu \nu}+\frac{1}{2} m_{A^{\prime}}^{2} A_{\mu}^{\prime 2},
$$

where $m_{A^{\prime}}$ is the dark photon mass and the dimensionless coupling, $\epsilon \ll 1$, controls the strength of kinetic mixing. DM coupled to a massless dark photon $\left(m_{A^{\prime}}=0\right)$ induces an effective millicharge $q_{\mathrm{eff}}=\epsilon e^{\prime} / e$, in addition to DM

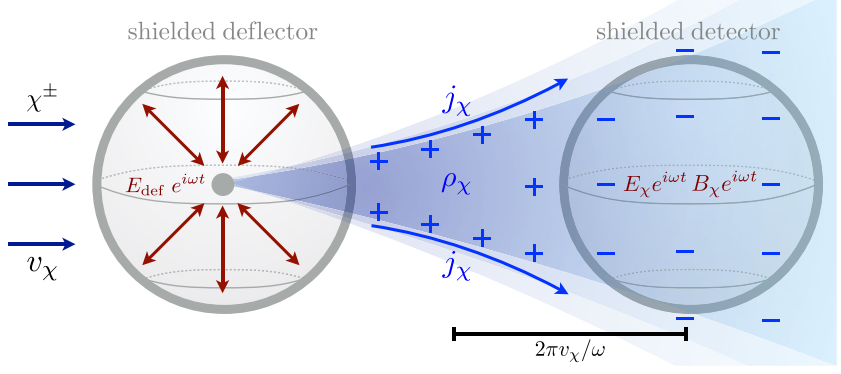

FIG. 1. Schematic of the experimental concept. Dark matter passing through an oscillating electric field is deflected, setting up propagating waves of alternating dark matter millicharge $\rho_{\chi}$ and millicurrent $j_{\chi}$ densities. Dark matter waves flow unimpeded through an electromagnetic shield, creating small electromagnetic fields that can be measured with a resonant $L C$ circuit inductively coupled to a magnetometer (not depicted) inside the shielded detector region. self-interactions controlled by $e^{\prime}$, where $e$ is the SM electric charge and $e^{\prime}$ is the dark photon gauge coupling. For nonzero $m_{A^{\prime}}$, DM interactions with SM matter are millichargelike over distance scales $\lesssim m_{A^{\prime}}^{-1}$ and exponentially screened at larger distances. We consider an experimental apparatus localized to $\mathcal{O}$ (meter)-scale distances, for which $m_{A^{\prime}}=0$ and $m_{A^{\prime}} \lesssim$ meter $^{-1} \sim 10^{-7} \mathrm{eV}$ are qualitatively indistinguishable. We focus on the massless case for simplicity and discuss finite-mass corrections in the Supplemental Material [16].

Sensitivity to this range of mediator masses is well matched to models of sub-MeV DM production in the early Universe. The primary benchmark model for production of sub-MeV DM is the "freeze-in" [2] of a DM abundance from the annihilations of thermal electrons [17-19] (and a related reaction, plasmon decay [20]). These reactions generate a DM abundance consistent with observations for couplings of size $q_{\mathrm{eff}} \sim 10^{-10} \times\left(m_{\chi} / \mathrm{keV}\right)^{-1 / 2}$ [20]. This region of parameter space is shown as the blue line in Fig. 2. In order to remain consistent with other constraints, realizing this scenario for sub-MeV dark photon mediators requires $m_{A^{\prime}} \lesssim$ $10^{-9} \mathrm{eV}[19,20]$. Therefore, viable freeze-in models for sub$\mathrm{MeV}$ DM lie firmly in the millichargelike regime for the class of experiments we consider. [It has been argued that millicharged DM may be evacuated from the galactic disk by supernova shocks [21,22] (but see, e.g., Ref. [23] for claims to the contrary). This effect is irrelevant for millichargelike DM with $m_{A^{\prime}} \gtrsim(100 \mathrm{pc})^{-1} \sim 10^{-25} \mathrm{eV}$, but might prevent all terrestrial experiments, including our concept, from detecting truly millicharged DM. For an investigation of other effects involving interactions between millicharged DM and galactic magnetic fields, see, e.g., Ref. [24].]

Overview of direct deflection.-A schematic illustration of the experimental setup is shown in Fig. 1. A chargesymmetric, spatially uniform DM population passes through a shielded region of radius $R$, with an electric field [Since virialized DM is nonrelativistic, millicharged DM is more efficiently deflected by electric (rather than magnetic) fields.] oscillating at angular frequency $\omega$. We refer to this region as the "deflector." The velocity distribution of DM in Earth's frame is expected to be approximately Maxwellian, shifted by a "wind" velocity, $v_{\text {wind }}$, from the Sun's motion in the Milky Way, with velocity dispersion $v_{0} \sim v_{\text {wind }} \sim \mathcal{O}(100) \mathrm{km} / \mathrm{s}$ [25].

As millicharged DM passes through the deflector, it is subject to an electric force that separates positively and negatively charged particles. This creates a propagating wave train of alternating millicharge $\left(\rho_{\chi}\right)$ and millicurrent $\left(j_{\chi}\right)$ densities of length $\sim 2 \pi v_{\text {wind }} / \omega$, which diffuse outwards due to dispersion in the DM velocity distribution. DM particles easily penetrate electromagnetic shielding, inducing small electromagnetic fields within the shielded detection region of Fig. 1. These fields have known oscillation frequency and phase, and can be measured using an electric field pickup antenna coupled to a resonant 


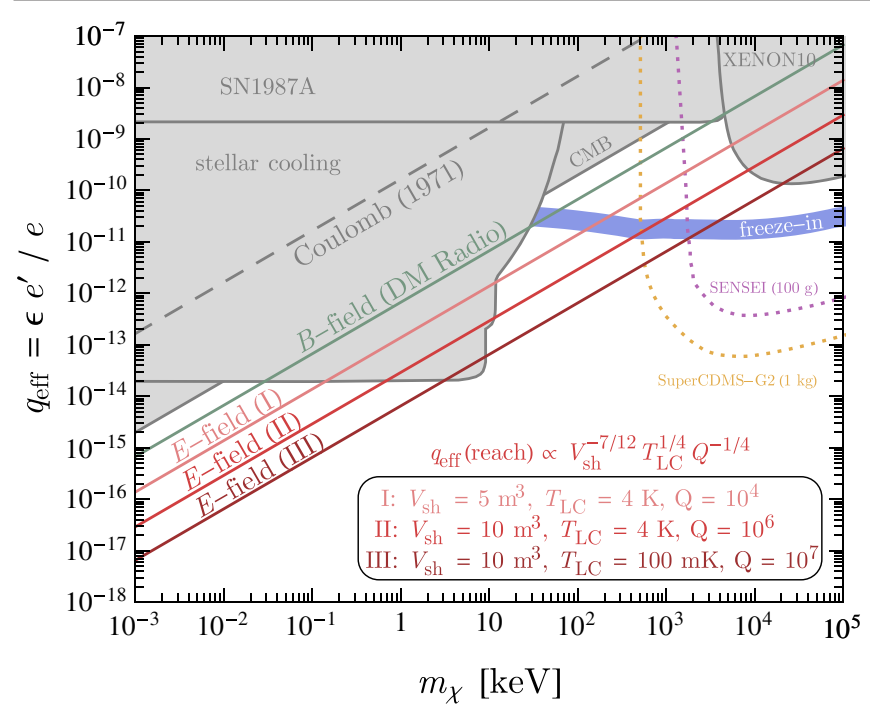

FIG. 2. The anticipated reach to millicharged dark matter in the $q_{\text {eff }}-m_{\chi}$ plane for various experimental configurations of our setup at $90 \%$ C.L., compared to existing constraints (shaded gray). In all cases, we assume a year of integration time, a spatially averaged field strength of $\left\langle E_{\text {def }}\right\rangle=10 \mathrm{kV} / \mathrm{cm}$, and $\omega=100 \mathrm{kHz}$. The green line corresponds to the projected reach of a detector optimized for detection of magnetic fields, such as the DM Radio experiment [6]. The reach of dedicated $L C$ resonators optimized for detecting electric fields is also shown. The lines labeled $E$-field (I-III) correspond to various deflector or shield volumes, $L C$ circuit temperatures, and quality factors as indicated in the legend. Also shown are the direct detection sensitivities of 1-year exposures for the near-term planned experiments SENSEI (100 g) (purple) [7,8] and SuperCDMSG2 (1 kg) (yellow) [1], assuming zero background. Longer-term $\mathrm{R} \& \mathrm{D}$ on direct detection concepts with meV-scale energy thresholds (such as detectors using superconductors [9], Dirac materials [10], or polar crystals [11,12] as targets) could extend direct detection sensitivity to $\mathrm{keV}$-scale DM masses. Along the solid blue line, the millicharge abundance from freeze-in production in the early Universe is in agreement with the observed dark matter energy density. We also show an estimate of a limit derived from a test of Coulomb's law as a dashed gray line $[13,14]$.

$L C$ circuit and SQUID amplifier. Relative to the DM wind (due to the Sun's galactocentric velocity), the apparatus rotates once per sidereal day; we have illustrated the geometry where the signal is maximized in Fig. 1.

Inducing dark matter waves.-Oscillating the sign of the deflector field allows for resonant read-out, but the oscillations should be slow enough that DM particles traverse the deflector within one period, i.e., $\omega \lesssim \pi v_{\chi} / R \sim \mathrm{MHz} \times(R / \text { meter })^{-1}$, where we take the characteristic DM velocity to be $v_{\chi} \sim v_{0 \text {,wind }} \sim 10^{-3} c$. When $\omega$ satisfies this upper bound, we can treat the fields as quasistatic. Moreover, astrophysical bounds on DM self-interactions constrain the Debye length of the DM plasma to be $\gtrsim 100$ meters for $m_{\chi} \gtrsim \mathrm{eV}$ [26], guaranteeing that collisional or backreaction effects in the DM plasma can be neglected when modeling the apparatus of Fig. 1.
In the Supplemental Material [16], we derive millicharge and millicurrent densities for general DM velocity distributions and deflector charge distributions. The DM millicharge density can be simply expressed in terms of general (multipole and trace) moments of the deflector charge distribution. For any deflector surrounded by a grounded shield, all multipole moments vanish (as required to obtain vanishing electric fields outside the shield). The induced DM charge density far outside the shield $(|\mathbf{x}| \gg R)$ is therefore dominated by the leading trace moment of the deflector-plus-shield charge distribution, which is its charge-radius-squared $\left(\mathcal{R}_{\text {def }}^{2}\right)$, i.e.,

$$
\rho_{\chi}(\mathbf{x}) \simeq-\frac{\left(e q_{\mathrm{eff}}\right)^{2} \rho_{\mathrm{DM}} \mathcal{R}_{\mathrm{def}}^{2}}{6 m_{\chi}^{2}} \int d v \nabla^{2} \frac{f(v \hat{\mathbf{x}})}{|\mathbf{x}|},
$$

where $\rho_{\mathrm{DM}} \simeq 0.4 \mathrm{GeV} / \mathrm{cm}^{3}$ is the local DM energy density, $f(\mathbf{v})$ is the DM velocity distribution in the lab frame (which we take to be a shifted-Maxwellian), and we have taken the deflector to be centered at the origin. The velocity integral in Eq. (2) scales with distance as $1 /|\mathbf{x}|^{3}$.

For a given enclosed deflector charge, $q_{\mathrm{def}}$, and spherical shield of radius $R$, a centered point charge maximizes the charge radius of the shielded deflection region and therefore represents the optimal deflector. The DM charge density induced by this geometry is shown in the Supplemental Material [16]. Similar DM charge densities are obtained for any shielded deflector with nonvanishing charge radius.

The distance scaling of Eq. (2) can be understood as follows: The millicharge distribution induced by an unshielded point charge deflector is approximately given by Debye screening,

$$
\rho_{\chi}^{\text {Debye }}(\mathbf{x}) \sim-\frac{\left(e q_{\mathrm{eff}}\right)^{2} \rho_{\mathrm{DM}} \phi_{\mathrm{def}}(\mathbf{x})}{m_{\chi}^{2} v_{0}^{2}},
$$

with $\phi_{\text {def }}(\mathbf{x}) \sim e q_{\text {def }} /|\mathbf{x}|$. The charge-radius squared that induces the leading $\rho_{\chi}$ for a shielded deflector [see Eq. (2)] is a second moment, and so the resulting form of $\rho_{\chi}$ must be further suppressed by $R^{2} /|\mathbf{x}|^{2}$. For $v_{0} \sim v_{\text {wind }}$, Eq. (2) is hence parametrically of the form

$$
\rho_{\chi}(\mathbf{x}) \sim \rho_{\chi}^{\text {Debye }}(R)(R /|\mathbf{x}|)^{3} .
$$

Since these DM charge densities travel in the lab frame at a speed $\sim v_{\text {wind }}$, the corresponding current densities are roughly, $j_{\chi}(\mathbf{x}) \sim \rho_{\chi}(\mathbf{x}) v_{\text {wind }}$. For the optimal geometry of Fig. 1, in which the DM wind is aligned with the detection region, these parametric expressions agree within $\mathcal{O}(1)$ factors with the detailed calculations presented in the Supplemental Material [16].

Detecting dark matter waves.-As shown in the setup of Fig. 1, to detect the DM millicharge or millicurrent 
densities, a detector is placed downstream of the deflector. The detector should be surrounded by its own electromagnetic shield, in order to reduce noise from external non-DM sources. We assume that the characteristic length scale of this shielded detection region is comparable to that of the deflector and is therefore also in the quasistatic limit. For concreteness, we take the detector shield to be a sphere of radius $R$.

The charge and current densities oscillate inside the detector shield at the same frequency as the deflector, $\rho_{\chi}(t) \simeq \rho_{\chi} e^{i \omega t}, \boldsymbol{j}_{\chi}(t) \simeq \boldsymbol{j}_{\chi} e^{i \omega t}$. Oscillating charge and current densities inside a conducting shield generate visible electric $\left(E_{\chi}\right)$ and magnetic $\left(B_{\chi}\right)$ fields that oscillate at the same frequency. In the quasistatic limit $(\omega \ll 1 / R)$, the dominant effect of the charge (current) density is a small electric (magnetic) field. We numerically calculate these fields inside the detector shield using the results for $\rho_{\chi}$ and $\boldsymbol{j}_{\chi}$ presented in the Supplemental Material [16]. Parametrically, we expect $E_{\chi} \sim \rho_{\chi} R$ and $B_{\chi} \sim j_{\chi} R$. The fact that $j_{\chi} \sim \rho_{\chi} v_{\chi}$ therefore implies that the electric field sourced by the DM charge density is velocity enhanced compared to the magnetic field sourced by the current density $\left(E_{\chi} \sim v_{\chi}^{-1} B_{\chi}\right)$, as expected for nonrelativistic charge carriers.

An excellent detector for these DM-sourced electromagnetic fields is a well-shielded resonant $L C$ circuit and antenna, inductively coupled to a SQUID. $L C$ circuits can operate at frequencies much smaller than the inverse geometric size of their corresponding circuit components $\left(\omega_{L C}=1 / \sqrt{L C} \ll 1 / R\right)$, in contrast to, e.g., superconducting rf cavities. (It is interesting to note that recent work has investigated the possibility that a subcomponent of the millicharged DM population could be accelerated to semirelativistic speeds by supernova remnants [21,23,27]. Depending on the abundance of such "dark cosmic rays," a setup similar to that shown in Fig. 1 involving a pair of resonant SRF cavities could have greatly enhanced sensitivity due to their extremely large field gradients and quality factors.) If the $L C$ circuit is resonantly matched to the deflector frequency $\left(\omega_{L C} \simeq \omega\right)$, then in the presence of the DM charge and current densities, the $L C$ circuit responds by ringing up the small oscillating fields over many cycles, quantified by its large quality factor, $Q \gg 1$. Such technology has been extensively developed and tested by AURIGA [28] to detect gravity waves and will be implemented by DM Radio [6] to search for ultralight coherent DM fields.

An $L C$ resonator with a large inductor, such as that proposed for the DM Radio experiment, couples mainly to the oscillating magnetic fields in the detector sourced by DM currents [6]. Detecting the parametrically larger electric field signals from DM charges motivates an $L C$ resonator incorporating a large capacitor or receiving antenna. [This is in contrast to the case of axion DM, where electric signals have been considered [29] but are suppressed relative to the magnetic one $[30,31]$.
The difference in scaling arises because in our setup, electromagnetic sources (millicharged DM particles) are present inside the shielded detection region.] In this work, we consider both inductively and capacitatively coupled detectors. We assume that stray electromagnetic noise is sufficiently well shielded that the experiment is limited by thermal (Johnson-Nyquist) noise in the detector, with a power spectral density equal to $4 R_{L C} T_{L C}$, where $R_{L C}$ and $T_{L C}$ are the resistance and temperature of the $L C$ resonator, respectively [32]. The shielding requirements are similar to those for light-shining-through-a-wall cavity experiments [3-5] or DM Radio [6].

The signal-to-noise ratio (SNR) is given by the corresponding ratio of power spectral densities. For an experiment limited by thermal noise and assuming an integration time $t_{\text {int }}$ that is much shorter than the phase-coherence time of the deflector, the SNR is approximately $[5,33]$

$$
\mathrm{SNR} \simeq \frac{\omega Q t_{\text {int }}}{4 T_{L C}} \int_{\operatorname{det}} d^{3} \mathbf{x}\left(E_{\chi}^{2} \quad \text { or } \quad B_{\chi}^{2}\right) \propto\left(\frac{q_{\mathrm{eff}}}{m_{\chi}}\right)^{4} .
$$

The integral of $E_{\chi}^{2}$ or $B_{\chi}^{2}$ is over an effective detector volume (the physical volume of a resonant capacitor or inductor, or the effective volume of a receiving antenna). In either case, these volumes are bounded by the total volume of the shielded detector cavity, $V_{\text {sh }}$. A receiving antenna pickup allows for the physical volume of the $L C$ circuit to be significantly smaller than $V_{\mathrm{sh}}$, giving a considerable advantage in terms of cooling power.

Expected reach.-The projected reach of our setup scales as [For deflector and detector shields of different volumes, the reach in Eq. (6) scales as $V_{\operatorname{def}}^{-1 / 6} V_{\operatorname{det}}^{-5 / 12}$.]

$$
q_{\mathrm{eff}} / m_{\chi} \propto V_{\mathrm{sh}}^{-7 / 12}\left\langle E_{\mathrm{def}}\right\rangle^{-1 / 2} T_{L C}^{1 / 4}\left(\omega t_{\mathrm{int}} Q\right)^{-1 / 4},
$$

which is mainly sensitive to the deflector or shield volume, $V_{\text {sh }}$, and the spatially averaged strength of the deflecting field $\left\langle E_{\text {def }}\right\rangle$. The estimated sensitivities for different experimental configurations are shown in Fig. 2, compared to existing constraints (shaded gray) [13,34-38] and the projected reach of near-term planned direct detection searches for energy deposition from DM scattering (dotted lines). In all cases, we assume a year of integration time and a deflector operating with a spatially averaged field strength of $\left\langle E_{\text {def }}\right\rangle=10 \mathrm{kV} / \mathrm{cm}$ at a frequency of $\omega=100 \mathrm{kHz}$. Thermal voltage fluctuations due to Johnson-Nyquist noise are randomly distributed according to a Gaussian centered at zero. Since this implies that power fluctuations follow a rescaled chi-squared distribution, we estimate the thermal noise limited reach of an $L C$ resonator by requiring SNR $\gtrsim 2.7$, corresponding to a $90 \%$ confidence limit.

The green line in Fig. 2 shows the projected reach of the planned DM Radio experiment, which is optimized to detect small magnetic fields and is expected to consist of a meter ${ }^{3}$ detector with $T_{L C}=10 \mathrm{mK}$ and $Q=10^{6}$, assuming that it is modified to include a meter ${ }^{3}$ upstream 
deflector. The construction of thermal-noise-limited resonant $L C$ circuits operating at $\mathrm{kHz}$ frequencies with quality factors of $Q \simeq 10^{6}$ has already been firmly established by the existing gravity-wave experiment, AURIGA [39-41]. Therefore, we also consider a future dedicated experimental configuration optimized for measuring the electric field signal, as shown by the lines labeled " $E$ field (I-III)" in Fig. 2. These correspond to deflector or shield volumes, $L C$ circuit temperatures, and quality factors ranging from $V_{\mathrm{sh}}=(5-10)$ meter $^{3}, T_{L C}=100 \mathrm{mK}-4 \mathrm{~K}$, and $Q=$ $10^{4}-10^{7}$, as noted in the legend of Fig. 2. In particular, the $E$-field (I) configuration demonstrates that even a conservative setup could attain impressive sensitivity to cosmologically motivated parameter space. Compared to AURIGA and DM Radio, our setup does not need to scan or operate at frequencies below a $\mathrm{kHz}$, which often necessitates the use of lossy dielectrics. Hence, quality factors greater than $10^{6}$ might be more easily attainable.

In Fig. 2, we have assumed that the separation distance between the deflector and detector is small compared to their overall size [a geometrical penalty as in Eq. (4) still exists because of the finite sizes of the deflector and detector]. We note that spherical geometries, such as that used in Ref. [13], can potentially overcome this minor penalty. We have also time averaged the power in Eq. (5) over a sidereal day, assuming that the axis joining the deflector and detector regions of Fig. 1 is aligned with the direction of the DM wind at time $t=0$ (see the discussion in the Supplemental Material [16] and in, e.g., Ref. [12]). The directionality of the DM wind introduces an $\mathcal{O}(1)$ fractional daily modulation to the signal as it follows the rotation of Earth, which provides an additional handle to discriminate a signal from unforeseen systematics or noise. This is discussed in more detail in the Supplemental Material [16].

Discussion.-A unique qualitative feature of the direct deflection technique discussed here is that the sensitivity improves at smaller masses, corresponding to larger DM number densities and smaller momentum carried by individual DM particles. Probing the collective effects of the large number density of DM particles, instead of relying on the energy deposition from a single DM scattering event, allows for $L C$ resonators coupled to electromagnetic deflectors to probe parameter space for DM masses well below the $\mathrm{keV}$ scale. We note that cosmological and astrophysical constraints have not yet been extensively investigated for such small masses; we leave the detailed investigation of these effects to future work.

We also note that our classical calculations are not valid to arbitrarily small DM masses. For $m_{\chi} \lesssim 0.1 \mathrm{meV}$, the de Broglie wavelength of an incoming DM particle is $\left(m_{\chi} v_{\chi}\right)^{-1} \gtrsim \mathcal{O}$ (meter), and hence comparable to the deflector size. In this regime (not shown in Fig. 2), a quantum treatment of millicharge and millicurrent production is required.

Our setup highlights the ability to detect DM in the Milky Way halo by inducing collective disturbances into the DM population through the manipulation of strong electromagnetic fields in the lab. Similar techniques can be more generally applied beyond what is considered in this work. Such ideas include, e.g., the application of specifically engineered field configurations to accelerate, focus, or trap DM near standard direct detection scattering targets or resonant detectors or using similar "deflection-detection" approaches to search for alternative types of DM-SM interactions. We leave such considerations to future work.

We thank Tony Beukers, Saptarshi Chaudhuri, Paolo Falferi, Peter Graham, Yoni Kahn, Tongyan Lin, Michael Peskin, Tor Raubenheimer, Jesse Thaler, Andrea Vinante, and Marco Zanetti for valuable discussions. The authors are supported by the U.S. Department of Energy under Contract No. DE-AC02-76SF00515.

[1] M. Battaglieri et al., U.S. Cosmic Visions: New Ideas in Dark Matter College Park, MD, USA, 2017 (2017).

[2] L. J. Hall, K. Jedamzik, J. March-Russell, and S. M. West, J. High Energy Phys. 03 (2010) 080.

[3] J. Jaeckel and A. Ringwald, Phys. Lett. B 659, 509 (2008).

[4] M. Betz, F. Caspers, M. Gasior, M. Thumm, and S. W. Rieger, Phys. Rev. D 88, 075014 (2013).

[5] P. W. Graham, J. Mardon, S. Rajendran, and Y. Zhao, Phys. Rev. D 90, 075017 (2014).

[6] M. Silva-Feaver et al., IEEE Trans. Appl. Supercond. 27, 1 (2017).

[7] M. Crisler, R. Essig, J. Estrada, G. Fernandez, J. Tiffenberg, M. S. Haro, T. Volansky, and T.-T. Yu (SENSEI Collaboration), Phys. Rev. Lett. 121, 061803 (2018).

[8] O. Abramoff et al. (SENSEI Collaboration), Phys. Rev. Lett. 122, 161801 (2019).

[9] Y. Hochberg, M. Pyle, Y. Zhao, and K. M. Zurek, J. High Energy Phys. 08 (2016) 057.

[10] Y. Hochberg, Y. Kahn, M. Lisanti, K. M. Zurek, A. G. Grushin, R. Ilan, S. M. Griffin, Z.-F. Liu, S. F. Weber, and J. B. Neaton, Phys. Rev. D 97, 015004 (2018).

[11] S. Knapen, T. Lin, M. Pyle, and K. M. Zurek, Phys. Lett. B 785, 386 (2018).

[12] S. Griffin, S. Knapen, T. Lin, and K. M. Zurek, Phys. Rev. D 98, 115034 (2018).

[13] E. R. Williams, J. E. Faller, and H. A. Hill, Phys. Rev. Lett. 26, 721 (1971).

[14] S. Mitra, Phys. Rev. D 74, 043532 (2006).

[15] B. Holdom, Phys. Lett. 166B, 196 (1986).

[16] See Supplemental Material at http://link.aps.org/ supplemental/10.1103/PhysRevLett.124.011801 for we first give a detailed derivation of the dark matter charge and current densities. In the later sections, we discuss daily modulation of the signal, and then investigate the dark matter-induced electromagnetic fields for massive dark photons and show that this reduces to the simple massless case when the dark photon is much lighter than the inverse geometric size of the experimental setup.

[17] R. Essig, J. Mardon, and T. Volansky, Phys. Rev. D 85, 076007 (2012). 
[18] X. Chu, T. Hambye, and M. H. G. Tytgat, J. Cosmol. Astropart. Phys. 05 (2012) 034.

[19] S. Knapen, T. Lin, and K. M. Zurek, Phys. Rev. D 96, 115021 (2017).

[20] C. Dvorkin, T. Lin, and K. Schutz, Phys. Rev. D 99, 115009 (2019).

[21] L. Chuzhoy and E. W. Kolb, J. Cosmol. Astropart. Phys. 07 (2009) 014.

[22] S. D. McDermott, H.-B. Yu, and K. M. Zurek, Phys. Rev. D 83, 063509 (2011).

[23] D. Dunsky, L. J. Hall, and K. Harigaya, J. Cosmol. Astropart. Phys. 07 (2019) 015.

[24] A. Stebbins and G. Krnjaic, J. Cosmol. Astropart. Phys. 12 (2019) 003.

[25] K. Freese, M. Lisanti, and C. Savage, Rev. Mod. Phys. 85, 1561 (2013).

[26] T. Lin, Proc. Sci., 333 (2019) 009.

[27] P.-K. Hu, A. Kusenko, and V. Takhistov, Phys. Lett. B 768, 18 (2017).

[28] M. Cerdonio et al., Classical Quantum Gravity 14, 1491 (1997).

[29] B. T. McAllister, M. Goryachev, J. Bourhill, E. N. Ivanov, and M. E. Tobar, arXiv:1803.07755.
[30] J. Ouellet and Z. Bogorad, Phys. Rev. D 99, 055010 (2019).

[31] M. Beutter, A. Pargner, T. Schwetz, and E. Todarello, J. Cosmol. Astropart. Phys. 02 (2019) 026.

[32] H. Nyquist, Phys. Rev. 32, 110 (1928).

[33] D. Budker, P. W. Graham, M. Ledbetter, S. Rajendran, and A. O. Sushkov, Phys. Rev. X 4, 021030 (2014).

[34] R. Essig, A. Manalaysay, J. Mardon, P. Sorensen, and T. Volansky, Phys. Rev. Lett. 109, 021301 (2012).

[35] R. Essig, T. Volansky, and T.-T. Yu, Phys. Rev. D 96, 043017 (2017).

[36] H. Vogel and J. Redondo, J. Cosmol. Astropart. Phys. 02 (2014) 029.

[37] J. H. Chang, R. Essig, and S. D. McDermott, J. High Energy Phys. 09 (2018) 051.

[38] E. D. Kovetz, V. Poulin, V. Gluscevic, K. K. Boddy, R. Barkana, and M. Kamionkowski, Phys. Rev. D 98, 103529 (2018).

[39] L. Baggio et al., Phys. Rev. Lett. 94, 241101 (2005).

[40] M. Bonaldi, P. Falferi, R. Dolesi, M. Cerdonio, and S. Vitale, Rev. Sci. Instrum. 69, 3690 (1998).

[41] M. Bonaldi, P. Falferi, M. Cerdonio, A. Vinante, R. Dolesi, and S. Vitale, Rev. Sci. Instrum. 70, 1851 (1999). 